THE X-RAY ZURICH ENVIRONMENTAL STUDY (X-ZENS). II.

$X$-RAY OBSERVATIONS OF THE DIFFUSE INTRAGROUP

MEDIUM IN GALAXY GROUPS

Miniati, Francesco

2016-03-01

Miniati , F , Finoguenov , A , Silverman , J D , Carollo , M , Cibinel , A , Lilly , S J \&

Schawinski , K 2016 , ' THE X-RAY ZURICH ENVIRONMENTAL STUDY (X-ZENS). II.

$X$-RAY OBSERVATIONS OF THE DIFFUSE INTRAGROUP MEDIUM IN GALAXY

GROUPS ' , Astrophysical Journal , vol. 819 , no. 1 , 26 . https://doi.org/10.3847/0004-637X/819/1/26

http://hdl.handle.net/10138/183631

https://doi.org/10.3847/0004-637X/819/1/26

unspecified

publishedVersion

Downloaded from Helda, University of Helsinki institutional repository.

This is an electronic reprint of the original article.

This reprint may differ from the original in pagination and typographic detail.

Please cite the original version. 


\title{
THE X-RAY ZURICH ENVIRONMENTAL STUDY (X-ZENS). II. X-RAY OBSERVATIONS OF THE DIFFUSE INTRAGROUP MEDIUM IN GALAXY GROUPS
}

\author{
Francesco Miniati $^{1}$, Alexis Finoguenov ${ }^{2}$, John D. Silverman ${ }^{3}$, Marcella Carollo ${ }^{1}$, \\ Anna Cibinel ${ }^{4}$, Simon J. Lilly ${ }^{1}$, and Kevin Schawinski ${ }^{1}$ \\ ${ }^{1}$ Physics Department, Wolfgang-Pauli-Strasse 27, ETH-Zürich, CH-8093, Zürich, Switzerland; fm@phys.ethz.ch \\ ${ }^{2}$ Department of Physics, Gustaf Hällströmin katu 2a, FI-00014, University of Helsinki, Finland \\ ${ }^{3}$ Institute for the Physics and Mathematics of the universe (IPMU), University of Tokyo, Kashiwanoha 5-1-5, Kashiwa-shi, Chiba 277-8568, Japan \\ ${ }^{4}$ Astronomy Center, Dept. of Physics and Astronomy, University of Sussex, Falmer, Brighton BN1 9QH, UK \\ Received 2014 June 22; accepted 2016 January 10; published 2016 February 24
}

\begin{abstract}
We present the results of a pilot XMM-Newton and Chandra program aimed at studying the diffuse intragroup medium (IGM) of optically selected nearby groups from the Zurich ENvironmental Study (ZENS) catalog. The groups are in a narrow mass range about $10^{13} M_{\odot}$, a mass scale at which the interplay between the IGM and the group member galaxies is still largely unprobed. X-ray emission from the IGM is detected in the energy band $0.5-2 \mathrm{keV}$ with flux $\leqslant 10^{-13} \mathrm{erg} \mathrm{s}^{-1} \mathrm{~cm}^{-2}$, which is one order of magnitude fainter than for typical ROSAT groups (RASS). For many groups, we set upper limits on the X-ray luminosity, indicating that the detections are likely probing the upper envelope of the X-ray emitting groups. We find that weighting the group halo mass by the fraction of the total stellar mass locked in the bulge galaxy components might reduce the bias of mass estimates based on the total optical luminosity with respect to the X-ray mass estimates, (consistent with Andreon, at larger mass scales). We measure a stellar mass fraction with a median value of about $1 \%$, with a contribution from the most massive galaxies between $30 \%$ and $50 \%$. Optical and X-ray data often give complementary answers concerning the dynamical state of the groups, and are essential for a complete picture of the group system. Extending this pilot program to a larger sample of groups is necessary to unveil any imprint of interaction between member galaxies and IGM in halo potentials of key importance for environmentally driven galactic evolution.
\end{abstract}

Key words: galaxies: groups: general - large-scale structure of universe - methods: observational - X-rays: general

\section{INTRODUCTION}

Galaxy groups in the mass range $10^{13}-10^{14} M_{\odot}$ are key structures in the cosmic fabric. Containing typically between a few to a few tens of member galaxies (Mulchaey 2000; Mulchaey et al. 2003), they are significantly more numerous than galaxy clusters, trace the filamentary components of the large-scale structure (e.g., Eke et al. 2004a) and are likely to contain a large fraction of the unaccounted cosmic baryons (Fukugita et al. 1998). Groups have historically been detected in the X-ray emission, typically extending on scales of a few hundred kiloparsecs, revealing a gas in a state of diffuse, ionized, and metal-rich plasma with temperature around one $\mathrm{keV}$. The plasma emission mechanism is a combination of thermal bremsstrahlung and line emission, the latter more prominent in groups than in clusters of galaxies due to the lower plasma temperature. The detection of hot plasma bears witness to the fact that groups are not simply associations of galaxies by way of projection effects, but real physical systems, gravitationally bound and having undergone some degree of virialization.

Groups host about $50 \%-70 \%$ of today's $L *$ galaxies (Eke et al. 2005), thus providing the environment most commonly experienced by the latter. The role and impact of a group environment on galaxies is therefore the subject of detailed studies in both optical and X-ray surveys (e.g., Rasmussen et al. 2006; Weinmann et al. 2006; Poggianti et al. 2008; McGee et al. 2011; Giodini et al. 2012; Knobel et al. 2012; Carollo et al. 2013, among the others). Theoretical models indicate that groups are instrumental in triggering processes that accelerate galaxy evolution. For example, the in-spiral timescale of dynamical friction varies in proportion to $\sigma^{3} / \rho$, where $\sigma$ and $\rho$ are the halo velocity dispersion and density, respectively. Thus, compared to the field and galaxy clusters, galaxy tidal interactions due to close encounters (harassment) and mergers take place on a cosmologically short timescale in groups, characterized by significant over-density and relatively low velocity dispersion (Barnes 1990a). Such galaxy-galaxy interactions induce dynamical instabilities (Mayer et al. 2001), plausibly allowing for the fueling of central super-massive black holes (Barnes \& Hernquist 1991; Barnes 2002; Mihos et al. 2003) and are likely to play a major role in the evolution of massive galaxies within the group environment (McIntosh et al. 2008; Feldmann et al. 2010; Robotham et al. 2013). In addition, gas dynamical interactions with the diffuse intragroup medium (IGM), such as ram-pressure stripping of cold gas in the stellar disk and/or strangulation of the baryonic supply from the halo reservoir, can severely reduce the gas available for star formation and feedback processes even in relatively low-density environments (Kawata \& Mulchaey., 2008; McCarthy et al. 2008), possibly leading to the observed satellite quenching in groups (e.g., van den Bosch et al. 2008; Skibba 2009; Peng et al. 2012; Wetzel et al. 2013; Carollo et al. 2014; Tal et al. 2014).

The group mass sets an important scale, one at which the processes of large-scale structure formation and galaxy formation meet. In fact, the thermodynamic properties of the IGM deviate substantially from scaling relations obeyed by massive clusters, an indication that processes other than gravity and (magneto-) hydrodynamics affect in an important way the gas energetics at this mass scale. Particular attention has been devoted to the study of gas entropy, because it is conserved during adiabatic processes and changes only according to the 
net amount of thermal energy either absorbed or released by the gas. Gas entropy in groups appears to be in clear excess with respect to the expected value from the scaling relations obeyed by clusters, (Ponman et al. 1999, 2003; Lloyd-Davis et al. 2000; Finoguenov et al. 2001; Mahdavi et al. 2005; Prat et al. 2010), a fact ascribed, at least in part, to energetic feedback from galaxy formation processes (e.g., Cavaliere et al. 1998; Borgani et al. 2005; Voit et al. 2005; Giodini et al. 2010; McCarthy et al. 2010). In fact, due to the group's shallow potential well and corresponding low virial temperature, the energy released by past star formation and active galactic nucleus (AGN) activity leaves a distinct footprint on the thermodynamic properties of the IGM. In addition, the IGM appears highly enriched with significant amounts of metals, most likely transported there from the parent galaxy through stellar winds (Rasmussen \& Ponman 2009; McCarthy et al. 2010). Winds associated with metal transition lines observed at redshift $z \sim 1$ appear to be strongly magnetized (Bernet et al. 2008, 2010, 2012), so the IGM is likely to be significantly magnetized as well, in addition to being metal rich.

The above interplay between galaxies and IGM is important because it affects the observational properties of X-ray groups as well as those of galaxies. Its study is important in shedding light on the origin of the observed physical properties of the IGM, including, among others, the radial profile of thermodynamic quantities such as entropy, temperature, and their dependence with virial mass (or any of its proxies). Likewise, the feedback processes described above, are believed to play a crucial role in galaxy evolution. In particular, current models suggest that the growth of galaxies is intimately related to the growth of their black holes, and that AGN activity is key in preventing excessive cooling in massive galaxy halos (Bower et al. 2006, 2008; Croton et al. 2006).

From an observational point of view, the study of the interplay between galaxies and the IGM remains incomplete for two basic reasons. First, it is now well known that groups are a heterogeneous class, spanning a wide range in dynamical state (Mulchaey 2000) and X-ray emission (Ekcmiller et al. 2012). Because X-ray observations provide the most straightforward way of identifying groups, X-ray selected group samples are traditionally the best studied (e.g., Heldson \& Ponman 2000; Finoguenov et al. 2001; Osmond \& Ponman 2004; Mahdavi et al. 2005; Rasmussen \& Ponman 2007), and have delivered many important results. However, dynamically relaxed groups tend to be more X-ray bright, which raises an important and well appreciated issue concerning the representative character of X-ray selected group samples. At cluster scales, several studies have indeed shown that the X-ray properties of optically selected galaxy clusters differ substantially from those of the X-ray-selected structures (Donahue et al. 2002; Gilbank et al. 2004; Lubin et al. 2004; Popesso et al. 2007), however, most of the difference can be simply attributed to the role cool cores play in the X-ray selection of clusters. With the advent of large spectroscopic surveys, e.g., the SDSS (York et al. 2000) and the 2dFGRS (Colless et al. 2001), it has also become possible to identify group-sized structures spectroscopically, i.e., through the identification of their member galaxies. Pioneering studies on a few systems have demonstrated the existence, also at the mass scales of groups, of less relaxed structures with low-X-ray emissivity (Mulchaey 2000; Rasmussen et al. 2006) that were missed in shallow X-ray surveys (RASS).

As for the second reason for the above incompleteness, the study of galaxy groups up to now has been limited by the lack of accurate information about the properties of the member galaxies together with the X-ray data. It is now clear, however, that in view of the rich variety of properties of galaxies observed at fixed group mass scale as a function of galaxy mass and of rank within the groups as central or satellite (Weinmann et al. 2006, 2008; Carollo et al. 2013, 2014), this information is essential in order to elucidate the relation between group environment and the evolution of the member galaxies.

Earlier efforts to study X-ray representative samples of groups based on the optical selection of member galaxies (Ebeling et al. 1994; Burns et al. 1996; Ponman et al. 1996; Mahdavi et al. 1997, 2000), however, have been mostly based on shallow exposure RASS data and/or on limited information about the physical properties of member galaxies. More recently, the Complete Local Group Sample (CLoGS; O'Sullivan et al. 2014) project is attempting to produce the first statistically complete survey of galaxy groups observed in the X-ray, optical, and radio wavebands, which should greatly contribute to the investigation of the scientific questions raised above. Using a complementary approach, we have also started the Zurich ENvironmental Study (ZENS; Carollo et al. 2013, 2014; Cibinel et al. 2013a, 2013b), a program to build a representative sample of galaxy groups, unbiased with respect to dynamical conditions, with multi-wavelength coverage, and with fully determined properties of the member galaxies. The aim of our program is to study the environmental impact on the evolution of galaxies, including, among others, the interplay between galaxy formation and IGM. The ZENS project, described in more detail in Section 2, differs in several respects from CLoGs, which is also briefly discussed there for comparison.

In this paper, we report on initial efforts to study the IGM with X-ray observations of the ZENS groups. In particular, we present data taken during the past few years with the $X M M$ Newton and Chandra telescopes on a sub-sample of ZENS groups. In this paper, we focus on the diffuse X-ray emission while we report on the X-ray point-sorce detections in a companion paper (Silverman et al. 2014) aimed at investigating the role of AGNs in the context of galaxy evolution in groups.

The XMM-Newton and Chandra observations, including the data analysis, are described in Section 3, while results concerning the diffuse X-ray emission are presented in Section 4. Results and conclusions are summarized in Section 5. In the following, we assume a $\Lambda$-CDM universe with parameters $\Omega_{m}=0.2792, \Omega_{b}=0.0462, \Omega_{\Lambda}=1-\Omega_{m}$, and Hubble constant $H_{0}=70 \mathrm{~km} \mathrm{~s}^{-1} \mathrm{Mpc}^{-1}$ (Komatsu et al. 2009).

\section{THE ZENS AND SAMPLE SELECTION}

The ZENS project, including its design, the observations and the publicly available catalog, and all environmental, structure, and photometric measurements, are described in great detail in the first three papers of the ZENS series (Carollo et al. 2013; Cibinel et al. 2013a, 2013b, respectively Paper I, II and III), which we refer to, for further information. In the following, we summarize aspects of the project that are relevant to this paper.

The ZENS data set consists of 141 groups, containing a total of 1630 galaxies, randomly extracted from the complete sample 
of groups of the $2 \mathrm{dF}$ Percolation-inferred Galaxy Group catalog (Eke et al. 2004a; 2PIGG), with at least five cataloged members and within the narrow redshift range of $0.05<z<0.0585$.

The groups are classified in terms of total mass, dynamical state (relaxed or unrelaxed), and location within the large-scale structure environment, i.e., their proximity to massive clusters, filaments, or voids.

The ZENS galaxies have been fully characterized in terms of their stellar mass, star-formation activity, morphological/ structural properties, and central/satellite rank within the group (see Paper II and III). Resolved information such as size and strength of bars, color gradients, color maps (as well as bulge and disk colors), stellar masses and structure properties have also been derived for our ZENS sample. Among the structural/ photometric properties available for the ZENS galaxies, we will mostly focus on the bulge masses in this paper. Bulge masses were derived from the bulge+disk decomposition of the I- and B-band photometric data combined with mass-to-light ratios from the Bruzual \& Charlot (2003) stellar evolution models (see Paper III). In particular, we define the bulge fraction $f_{\text {bulge }}$ as the fraction of the stellar mass of group members with $M_{\text {galaxy }}>10^{10}$ that is locked in the bulge component, i.e.,

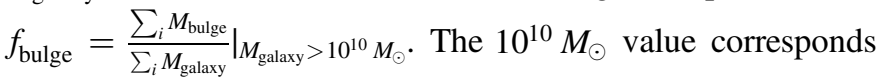
to the mass completeness limit for any spectral type in ZENS, effectively imposed by the quenched galaxies.

Among extant projects with similar objectives to ours, the already mentioned CLoGS is probably the one that comes closest in scope. In particular, the CLoGS project is aiming for a representative catalog of 53 local groups $(z<0.02)$ with optical, radio, and $X M M$ or Chandra coverage. Our ZENS study differs from this project in a number of important ways. The majority of the CLoGS groups (73\%) hosts a number of galaxies with $L_{B}>3 \times 10^{10.2} L_{\odot}$ equal to $R_{10.2}>3$. Only $20 \%$ of ZENS groups have such high richness parameters and have instead an average of $R_{10.2} \simeq 1-2$ (with a typical total membership of about 10 galaxies). The ZENS groups hence bracket smaller halo masses. In addition, the CLoGS group is selected to have at least one luminous $\left(L_{B}>3 \times 10^{10} L_{\odot}\right)$ early-type galaxy. While this likely ensures a group higher $\mathrm{X}$-ray luminosity, it is also limited to probing more massive systems. Furthermore, while a basic morphological classification and integrated photometric information is also available in the HYPERLEDA catalog (Paturel et al. 2003) from which CLoGS is based, the ZENS data set improves on such data by providing, as described above, a comprehensive set of detailed properties for the whole galaxies, their bulges, and disks.

The ZENS groups for which we carried out X-ray follow-up observations (either with XMM or Chandra) were selected to (1) have $>5$ spectroscopically confirmed members, (2) have halo masses in the range of a few $\times 10^{13} M_{\odot}$, (3) be representative of the entire ZENS group sample in terms of the galactic composition, and (4) fall within the $X M M$ or Chandra field of view (see also Silverman et al. 2014). The constraints on the number of galaxies and halo mass were imposed to exclude very small groups that are more strongly affected by uncertainties in the member identification (see Paper I) while still probing systems that are below the typical cluster mass. We did not impose any constraint on the expected $\mathrm{X}$-ray emission.

\section{OBSERVATIONS: DATA ACQUISITION, ANALYSIS, AND SOURCE DETECTION}

\subsection{XMM-Newton}

We have carried out XMM-Newton observations of ZENS groups during three consecutive observational programs (PI: Miniati, Prop. \#065530, Prop. \#067448, Prop. \#069374). As of today, nine observations of groups have been performed. We have also retrieved data for four additional groups from the archive, for a set of 13 XMM-Newton observations of our ZENS groups.

Details of the observations are presented in Table 1, which reports (1) the target name, (2) the observation ID, (3-4) the R.A. and decl. coordinates in J2000, (5) the group redshift, (6) the number of member galaxies, (7) the group mass inferred from the galaxy luminosity function, (8) XMM cycle of observation, (9) and the exposure time. The last column (10) contains notes about the observational data, in particular, whether the observations suffered from "flares." Note that columns (5)-(7) were extracted from the original Eke et al. (2004a, 2004b) catalogs.

The exposure times were estimated based on the expected $\mathrm{X}$-ray luminosity of our targets, in the range of $3 \times 10^{40}<L_{0.5-2 \mathrm{keV}} /\left(\mathrm{erg} \mathrm{s}^{-1}\right)<5 \times 10^{41} \quad$ (Rasmussen \& Ponman 2009). Detection of X-ray emission from groups is notoriously challenging. This is partly because X-ray luminosity scales as a total mass squared and becomes faint at the group mass scale, while studies of $z \ll 0.1$ extended sources are surface brightness limited even at total fluxes of $10^{-13} \mathrm{erg} \mathrm{s}^{-1} \mathrm{~cm}^{-2}$, which substantially reduces the sensitivity toward the low-mass halos at those redshifts. In addition, at such a mass scale, the X-ray luminosity-hydrostatic mass relation becomes characterized by considerable scatter (Ekcmiller et al. 2012), causing uncertainties in the observational forecast. While this reminds us of the risk of observational biases in X-ray-selected group samples, it also illustrates the difficulty associated with amending the problem.

Different exposure times in different observing cycles reflect different strategies underlying our successive proposals. In particular, in AO-9, we used long exposures because we aimed to measure the thermodynamic properties of the intragroup medium. This, however, was achieved only for one of the three observed groups, which led us to revise our strategy in AO-10, and split the program into two separate steps: first, carry out a short exposure, to determine the group luminosity and then follow up with deeper observations of bright enough groups so that their thermodynamic properties can be determined with reasonable exposure times. This approach is made difficult, however, by the large incidence of flares, which can completely corrupt the data for short exposure times. Slightly longer exposures were therefore employed in AO-11.

The standard processing of the $X M M$ observations includes the screening for the flares and making composite images from all three detectors in the $0.5-2$ and $2-7.5 \mathrm{keV}$ bands, excluding the energies affected by the strong instrumental lines, as discussed in Finoguenov et al. (2007). We only used 0.5-2 keV images for the detection of extended emission (the hard band is used to search for AGNs). For background subtraction and point source removal, we used the procedure of Finoguenov et al. (2007) with updates described in Bielby et al. (2010). We monitor the $\mathrm{S} / \mathrm{N}$ maps to control the quality of background removal. On several occasions, hot MOS1 and MOS2 chips 
Table 1

XMM-Newton's Log of ZENS Galaxy Group Observations

\begin{tabular}{|c|c|c|c|c|c|c|c|c|c|}
\hline Target & OBS ID & $\begin{array}{c}\text { R.A. } \\
(\mathrm{J} 2000)\end{array}$ & $\begin{array}{c}\text { decl. } \\
(\mathrm{J} 2000)\end{array}$ & Redshift $^{\mathrm{a}}$ & $\begin{array}{c}\text { \# Of } \\
\text { Galaxies }^{\mathrm{a}}\end{array}$ & $\begin{array}{c}M_{\text {Group }}{ }^{\text {a }} \\
\left(10^{13} M_{\odot}\right)\end{array}$ & Cycle & $\begin{array}{l}\text { Exp } \\
(\mathrm{ks})\end{array}$ & $\overline{\text { Note }^{b}}$ \\
\hline 2PIGG_s1520 & 0655300101 & 00:02:01.79 & $-34: 52: 55.5$ & 0.05434 & 9 & 1.55 & AO-9 & 33.0 & $\cdots$ \\
\hline 2PIGG_s1571 & 0655300301 & $02: 37: 04.33$ & $-25: 23: 34.3$ & 0.0568 & 10 & 1.52 & AO-9 & 38.8 & $\ldots$ \\
\hline 2PIGG_s1783 & 0655300601 & $22: 17: 26.33$ & $-36: 59: 48.1$ & 0.05833 & 8 & 4.90 & AO-9 & 39.4 & $\mathrm{~F}$ \\
\hline 2PIGG_s1614 & 0674480301 & $22: 25: 15.88$ & -252315.4 & 0.05676 & 18 & 4.98 & AO-10 & 15.5 & $\mathrm{~F}$ \\
\hline 2PIGG_s1471 & 0674480901 & $23: 45: 01.81$ & $-26: 37: 26.8$ & 0.05276 & 15 & 3.91 & AO-10 & 13.0 & $\ldots$ \\
\hline 2PIGG_n1466 & 0674480401 & 14:04:01.63 & $-01: 40: 06.9$ & 0.05292 & 17 & 4.980 & AO-10 & 15.0 & $\mathrm{~F}$ \\
\hline 2PIGG_n1572 & 0674480701 & $14: 25: 33.40$ & $-01: 30: 00.4$ & 0.05501 & 19 & 4.72 & AO-10 & 15.0 & $\cdots$ \\
\hline 2PIGG_s1799 & 0693741001 & $01: 14: 34.43$ & $-33: 56: 09.8$ & 0.05819 & 13 & 3.35 & AO-11 & 25.0 & $\mathrm{~F}$ \\
\hline 2PIGG_n1606 & 0693741601 & $10: 38: 36.50$ & $+01: 46: 01.2$ & 0.056 & 7 & 1.55 & AO-11 & 25.7 & \\
\hline 2PIGG_n1714 & 0150870401 & 10:36:06.57 & $-04: 02: 11.8$ & 0.0576 & 7 & 2.00 & AO-2 & 32.5 & A \\
\hline 2PIGG_n1377 & 0207060301 & $11: 32: 42.10$ & $-03: 50: 20.0$ & 0.05154 & 23 & 7.51 & AO-3 & 27.7 & $\mathrm{~A}, \mathrm{~F}$ \\
\hline 2PIGG_n1330 & 0305800101 & $10: 27: 36.72$ & $-03: 03: 58.8$ & 0.05044 & 5 & 1.02 & $\mathrm{AO}-4$ & 24.9 & $\mathrm{~A}$ \\
\hline 2PIGG_s1783 & 0550460801 & $22: 17: 26.33$ & $-36: 59: 48.1$ & 0.05833 & 8 & 4.90 & AO-7 & 28.0 & A \\
\hline
\end{tabular}

Notes.

${ }^{a}$ From Eke et al. (2004a, 2004b).

${ }^{\mathrm{b}} \mathrm{F}=$ flared, $\mathrm{A}=$ Archival Data.

Table 2

$X M M$-Newton Data on Diffuse Intragroup Emission

\begin{tabular}{|c|c|c|c|c|c|c|c|c|c|c|}
\hline Target & $\begin{array}{l}\text { Total } \\
\text { cnts }\end{array}$ & $\begin{array}{c}\text { Bkgd } \\
\text { cnts }\end{array}$ & $\begin{array}{c}\text { Point } \\
\text { cnts }\end{array}$ & $\begin{array}{c}\operatorname{Rad} \\
\left({ }^{\prime}\right)\end{array}$ & $\begin{array}{c}L_{\mathrm{x}}^{\mathrm{a}} \\
\left(10^{41} \mathrm{erg} \mathrm{s}^{-1}\right)\end{array}$ & $\begin{array}{c}M_{200} \\
\left(10^{13} M_{\odot}\right)\end{array}$ & $\begin{array}{c}R_{200} \\
\left({ }^{\prime}\right)\end{array}$ & $\begin{array}{c}T_{\mathrm{x}} \\
(\mathrm{keV})\end{array}$ & $\begin{array}{c}\text { Flux }^{\mathrm{b}} \\
\left(10^{-14} \mathrm{erg} / \mathrm{s} / \mathrm{cm}^{2}\right)\end{array}$ & $\mathrm{S} / \mathrm{N}$ \\
\hline 2PIGG_s1520 & $4707 \pm 69$ & 3576 & 608 & 3.3 & $3.38 \pm 0.50$ & $1.29 \pm 0.43$ & 7.56 & $0.44 \pm 0.11$ & $3.10 \pm 0.46$ & 6.77 \\
\hline 2PIGG_s1571 & $16938 \pm 130$ & 8534 & 1630 & 4.9 & $31.9 \pm 0.68$ & $4.96 \pm 1.65$ & 11.34 & $0.90 \pm 0.22$ & $27.88 \pm 0.59$ & 46.87 \\
\hline 2PIGG_s1614 & $2426 \pm 49$ & 2294 & 50 & 5.0 & $3.10 \pm 1.70$ & $1.23 \pm 0.37$ & 7.14 & $0.43 \pm 0.11$ & $2.57 \pm 1.41$ & $1.82^{\mathrm{d}}$ \\
\hline 2PIGG_s1471 & $3235 \pm 57$ & 2518 & 607 & 5.0 & $1.75 \pm 0.82$ & $0.87 \pm 0.23$ & 6.84 & $0.38 \pm 0.10$ & $1.63 \pm 0.76$ & $2.13^{\mathrm{d}}$ \\
\hline 2PIGG_n1572 & $3998 \pm 63$ & 3650 & 377 & 5.0 & $<1.69$ & $<0.85$ & $\cdots$ & $\cdots$ & $<1.43$ & $-0.93^{\mathrm{e}}$ \\
\hline
\end{tabular}

Notes.

a $0.1-2.4 \mathrm{keV}$.

${ }^{\mathrm{b}} 0.5-2 \mathrm{keV}$.

${ }^{\mathrm{c}}$ High residual background level.

d A 2 sigma detection, likely contaminated by unassociated emission.

e A 2 sigma-limit is calculated.

${ }^{\mathrm{f}}$ Upper limit is set by the level of residual background.

${ }^{\mathrm{g}}$ Unassociated emission.

were removed. The flux of detected point sources was removed using the point-spread function (PSF) model of XMM-Newton before proceeding with extended source detection, and the systematics of the process added to the errors (Finoguenov et al. 2009). Table 2 lists the contribution of the point sources to the total counts detected. The detection limit for AGN in the soft band corresponds to (1-3) $\times 10^{-15} \mathrm{erg} \mathrm{s}^{-1} \mathrm{~cm}^{-2}$ for XMM-Newton observations. This is deeper than, or comparable to, the statistical error on the flux determination. Thus, AGNs cannot contribute significantly to the detected fluxes. In case of available Chandra and XMM data for the same target we performed parallel analysis and compared the results, showing that estimated fluxes do not depend on the choice of the instrument.

After determining the genuinely diffuse component of the $\mathrm{X}$-ray flux, $F_{\mathrm{x}}$, we derive the X-ray luminosity in the (0.1-2.4) keV band, using the relation, $L_{(0.1-2.4) \mathrm{keV}}=4 \pi d_{L}^{2} K(z, T) F_{x} C(z, T)$, where $d_{L}(z)$ is the redshift dependent luminosity distance for the assumed cosmology, $K(z, T)$ is the $k$-correction and $C(z, T)$ is a factor converting the luminosity from the instrument band to the sought (0.1-2.4) keV band, based on a thermal emissivity model with a metallicity of 0.3 solar. The correction factor $C(z, T)$ depends on temperature, which is given by the $L_{(0.1-2.4) \mathrm{keV}}-T$ relation presented in Markevitch (1998) and extended for low temperature groups in Finoguenov et al. (2007), namely,

$$
T=T_{0}+T_{1}\left(\frac{L_{\mathrm{X}}}{E(z) L_{\mathrm{X}, 0}}\right)^{\alpha_{T}}
$$

with $T_{0}=0.2 \mathrm{keV}, T_{1}=6 \mathrm{keV}, L_{\mathrm{X}, 0}=10^{44.45} \mathrm{erg} \mathrm{s}^{-1}$, and $\alpha_{T}=1 / 2.1$. Thus, in practice, both X-ray luminosity and temperature are found simultaneously through an iterative procedure (Finoguenov et al. 2007). The values of the temperature inferred from the above relation are affected by an uncertainty of an order of 25\% (Markevitch 1998), which we consider to be the nominal error on the estimated 
Table 3

Chandra's Log of ZENS Galaxy Group Observations

\begin{tabular}{|c|c|c|c|c|c|c|c|c|c|}
\hline Target & OBS ID & $\begin{array}{c}\text { R.A. } \\
(\mathrm{J} 2000)\end{array}$ & $\begin{array}{c}\text { decl. } \\
(\mathrm{J} 2000)\end{array}$ & Redshift $^{\mathrm{a}}$ & $\begin{array}{c}\text { \# Of } \\
\text { Galaxies }^{\mathrm{a}} \\
\end{array}$ & $\begin{array}{c}M_{\text {Group }}{ }^{\mathrm{a}} \\
\left(10^{13} M_{\odot}\right)\end{array}$ & Cycle & $\begin{array}{l}\text { Exp } \\
(\mathrm{ks}) \\
\end{array}$ & Note \\
\hline 2PIGG_s1571 & 11613 & $02: 37: 04.33$ & $-25: 23: 34.3$ & 0.0568 & 10 & 1.52 & 11 & 10.06 & $\cdots$ \\
\hline 2PIGG_n1610 & $11617-11620$ & $09: 53: 38.23$ & $-05: 08: 21.4$ & 0.0562 & 10 & 1.45 & 11 & 10.03 & $\cdots$ \\
\hline 2PIGG_n1702 & $11621-11624$ & $09: 54: 30.67$ & $-04: 06: 03.3$ & 0.0574 & 9 & 2.26 & 11 & 9.88 & $\cdots$ \\
\hline 2PIGG_n1347 & 11625,11627 & 09:59:44.62 & $-05: 16: 52.6$ & 0.0521 & 10 & 2.90 & 11 & 10.29 & $\cdots$ \\
\hline 2PIGG_n1480 & 11629,11631 & $10: 15: 31.91$ & $-05: 37: 06.9$ & 0.0537 & 13 & 2.26 & 11 & 9.97 & $\cdots$ \\
\hline 2PIGG_n1320 & $11633-11636$ & $10: 17: 55.04$ & $-01: 22: 53.4$ & 0.0508 & 10 & 3.00 & 11 & 10.05 & $\cdots$ \\
\hline 2PIGG_n1441 & 11637,11639 & $11: 18: 10.68$ & $-04: 27: 36.1$ & 0.0531 & 15 & 3.41 & 11 & 9.97 & $\cdots$ \\
\hline 2PIGG_n1381 & $11641,11643,11644$ & $14: 28: 12.53$ & $-02: 31: 12.4$ & 0.0522 & 10 & 1.22 & 11 & 10.16 & $\cdots$ \\
\hline 2PIGG_n1598 & 11645 & $14: 35: 54.08$ & $-01: 16: 42.7$ & 0.0560 & 9 & 2.67 & 11 & 9.79 & $\cdots$ \\
\hline 2PIGG_n1746 & 11649,11652 & $14: 40: 20.07$ & $-03: 45: 56.2$ & 0.0585 & 9 & 1.65 & 11 & 10.18 & $\cdots$ \\
\hline 2PIGG_s1752 & 11653,11655 & $22: 21: 10.68$ & $-26: 00: 24.6$ & 0.0577 & 11 & 5.60 & 11 & 10.35 & $\cdots$ \\
\hline 2PIGG_s1671 & $11657-11660$ & $22: 24: 00.14$ & $-30: 00: 17.9$ & 0.0567 & 10 & 2.83 & 11 & 10.58 & $\cdots$ \\
\hline
\end{tabular}

Note.

${ }^{a}$ From Eke et al. (2004a, 2004b).

temperatures. The estimated temperature is used to infer the correction for the group flux left outside of the aperture.

Having determined the X-ray luminosity of the diffuse emission, we employ the scaling relation of Leauthaud et al. (2010) and its recent extension to low-mass groups presented in Finoguenov et al. (2015), to infer the mean halo virial mass, namely,

$$
M_{200}=\frac{A M_{0}}{E(z)}\left(\frac{L_{\mathrm{X}}}{E(z) L_{\mathrm{X}, 0}}\right)^{\alpha_{M_{200}}}
$$

with $E \approx 1.02, M_{0}=10^{13.7} h_{70}^{-1} M_{\odot}, L_{\mathrm{X}, 0}=10^{42.7} h_{70}^{-2} \mathrm{erg} \mathrm{s}^{-1}$, $A=1.35$, and $\alpha_{M_{200}}=0.6$. Note that the ZENs groups considered in this paper lie below the luminosity range of the groups used by Leauthaud et al. (2010) to determine the parameters $A$ and $\alpha_{M}$. The recent analysis of X-ray groups in the Extended Chandra Deep Field South of Finoguenov et al. (2015), however, shows that the above relations continue to hold in the mass and luminosity range of the ZENs groups. The relative uncertainty on the mass determination is of an order of $30 \%$ and this error on the mass will be considered in the analysis below. Finally, the over-density radius is trivially derived according to its definition

$$
R_{\Delta_{c}}=\left(\frac{3}{4 \pi} \frac{M_{\Delta_{c}}}{\Delta_{c} \rho_{c}}\right)^{\frac{1}{3}}
$$

where $\Delta_{c}=200,500$ is the over-density at which the mass is calculated and $\rho_{c}$ is the critical density for closure. The relative error is one-third times the relative error on the mass. Given their definition, these radii are merely a restatement of the corresponding mass.

Table 2 summarizes measurements of the X-ray emission from the diffuse IGM, including upper limits for the nondetections. In particular, it reports (1) target name, (2) total X-ray counts (extended and point sources including background), (3) background counts only, (4) counts due to point sources, (5) count extraction radius, (6) estimate of the total $\mathrm{X}$-ray luminosity in the rest frame $0.1-2.4 \mathrm{keV}$ band, and (7-9) corresponding virial mass, size, and temperature and corresponding uncertainties, as discussed above, except for the virial size, which is mostly provided for comparison with the extent of the count extraction radius, (10) the source flux extrapolated to the $R_{500}$ using the procedure outlined in Finoguenov et al. (2007), (11) signal-to-noise ratio. Note that the formal statistical significance of the detected emission is larger then the reported flux significance. The results based on these measurements are discussed in Section 4.

\subsection{Chandra}

We also carried out Chandra/ACIS-I observations of 12 ZENS galaxy groups in Cycle 11 (Prog. \#11700688; $120 \mathrm{ks}$ ). The observations were executed between 2009 September and 2010 October. Each target is observed for $10 \mathrm{ks}$, the main objective of these observations was the detection with at least four counts in the broad energy band $0.5-8 \mathrm{keV}$, of AGNs at $z \sim 0.05$ down to a limiting luminosity of $L_{(0.5-8 \mathrm{keV})} \sim 4 \times 10^{40} \mathrm{erg} \mathrm{s}^{-1}$. The field of view of ACIS-I $(16$ ! $9 \times 16$ !9; CCDs \#0-3) is sufficient to cover the sky area of these galaxy groups $\left(\right.$ size $\left.<10^{\prime}\right)$. The target positions were chosen to avoid having galaxies falling within or near chip gaps; this was accomplished by adjusting the pointing location once the planned observation date was set thus the roll angle was known. In Table 3, we provide details on the individual exposures, which is analogous to Table 1 for the XMM-Newton observations.

We employ a method to detect extended emission in our Chandra observations similar to that of a number of recent analyses (e.g., Cappelluti et al. 2012; Tanaka et al. 2013). We screen the event file by removing time intervals affected by flares, using the CIAO lc_clean tool. Exposure maps (effective area versus sky position) are generated for each observation listed in Table 3 using mkinstmap and mkexpmap. To do so, we generate an instrument map (effective area versus detector position) in the $0.5-2 \mathrm{keV}$ band using as a model spectrum to compute the sensitivity (i.e., a model weighing scheme) a power-law distribution with an index of 1.7. For our purposes, the difference in the final exposure map when using either a power-law or thermal spectrum is negligible. Using the merged event files, we generate a combined image for each galaxy group in the $0.1-2.4 \mathrm{keV}$ band.

The detection of diffuse emission requires the removal of point sources. To do so, we run "wvdecomp" (Vikhlinin et al. 
Table 4

Chandra Data on Diffuse Intragroup Emission

\begin{tabular}{|c|c|c|c|c|c|c|c|c|c|c|}
\hline Target & $\begin{array}{l}\text { Total } \\
\text { cnts }\end{array}$ & $\begin{array}{l}\text { Bkgd } \\
\text { cnts }\end{array}$ & $\begin{array}{l}\text { Point } \\
\text { cnts }\end{array}$ & $\begin{array}{c}\operatorname{Rad} \\
\left({ }^{\prime}\right)\end{array}$ & $\begin{array}{c}L_{\mathrm{x}}^{\mathrm{a}} \\
\left(10^{41} \mathrm{erg} \mathrm{s}^{-1}\right)\end{array}$ & $\begin{array}{c}M_{200} \\
\left(10^{13} \mathrm{M}_{\odot}\right)\end{array}$ & $\begin{array}{l}R_{200} \\
\quad\left({ }^{\prime}\right)\end{array}$ & $\begin{array}{c}T_{\mathrm{x}} \\
(\mathrm{keV})\end{array}$ & $\begin{array}{c}\text { Flux }^{\mathrm{b}} \\
\left(10^{-14} \mathrm{erg} / \mathrm{s} / \mathrm{cm}^{2}\right)\end{array}$ & $\mathrm{S} / \mathrm{N}$ \\
\hline 2PIGG_s1571 & $616 \pm 24.8$ & 332 & 17 & 3 & $34.33 \pm 3.19$ & $5.19 \pm 1.73$ & 11.4 & $0.93 \pm 0.23$ & $30.0 \pm 2.79$ & 10.76 \\
\hline 2PIGG_n1610 & $185 \pm 13.6$ & 179 & 28.9 & 2 & $<3.72$ & $<1.37$ & .. & $\ldots$ & $<3.20$ & $-1.68^{c}$ \\
\hline 2PIGG_n1702 & $86.9 \pm 9.3$ & 65 & 0 & 1.5 & $2.84 \pm 1.26$ & $1.16 \pm 0.39$ & 7.2 & $0.42 \pm 0.11$ & $2.29 \pm 1.02$ & $2.26^{\mathrm{d}}$ \\
\hline 2PIGG_n1347 & $194 \pm 13.9$ & 133 & 34.6 & 2 & $<3.26$ & $<1.27$ & $\ldots$ & $\ldots$ & $<3.24$ & $1.90^{\mathrm{c}}$ \\
\hline 2PIGG_n1480 & $157 \pm 12.5$ & 151 & 7.6 & 2 & $<3.07$ & $<1.22$ & $\ldots$ & $\ldots$ & $<2.86$ & $-0.13^{\mathrm{c}}$ \\
\hline 2PIGG_n1320 & $133 \pm 11.5$ & 68 & 14.7 & 1.4 & $5.39 \pm 1.24$ & $1.71 \pm 0.57$ & 9.0 & $0.50 \pm 0.13$ & $5.86 \pm 1.34$ & 4.36 \\
\hline 2PIGG_n1441 & $199 \pm 14.1$ & 155 & 20.1 & 2 & $<3.46$ & $<1.31$ & $\ldots$ & $\ldots$ & $<3.32$ & $1.70^{\mathrm{c}}$ \\
\hline 2PIGG_n1381 & $208 \pm 14.4$ & 159 & 37.8 & 2 & $<3.18$ & $<1.25$ & $\cdots$ & $\ldots$ & $<3.16$ & $0.78^{c}$ \\
\hline 2PIGG_n1598 & $184 \pm 13.6$ & 141 & 23.7 & 2 & $<3.69$ & $<1.36$ & $\cdots$ & $\cdots$ & $<3.19$ & $1.42^{\mathrm{c}}$ \\
\hline 2PIGG_n1746 & $62 \pm 7.9$ & 31 & 0 & 0.9 & $5.08 \pm 1.29$ & $1.65 \pm 0.55$ & 7.8 & $0.49 \pm 0.12$ & $4.09 \pm 1.04$ & 3.92 \\
\hline 2PIGG_s1752 & $374 \pm 19.3$ & 372 & 16 & 3 & $<5.16$ & $<1.66$ & $\cdots$ & $\ldots$ & $<4.27$ & $-0.72^{\mathrm{c}}$ \\
\hline 2PIGG_s1671 & $438 \pm 20.9$ & 391 & 27 & 3 & $<5.36$ & $<1.70$ & $\cdots$ & $\cdots$ & $<4.62$ & $0.96^{\mathrm{c}}$ \\
\hline
\end{tabular}

Notes.

a $0.1-2.4 \mathrm{keV}$.

b $0.5-2 \mathrm{keV}$.

c A $2 \sigma$ limit is calculated.

${ }^{\mathrm{d}}$ A $2 \sigma$ detection, likely contaminated by unassociated emission.

1998 ) on the $0.5-2.0 \mathrm{keV}$ band image with and $4 \sigma$ threshold. We remove the detected sources following the Chandra PSF model, as described in Finoguenov et al. (2009, 2015). The detection limit for an AGN in the soft band corresponds to $1 \times 10^{-15} \mathrm{erg} \mathrm{s}^{-1} \mathrm{~cm}^{-2}$, which is deeper than, or comparable to, the statistical error on the flux determination. Therefore, as in the case of XMM-Newton, AGNs cannot contribute significantly to the detected fluxes. The background X-ray emission is estimated using the procedure of Hickox \& Markevitch (2006) that uses the particle background (of nonastrophysical origin) measured from the stowed position of ACIS (http://cxc.cfa.harvard.edu/contrib/maxim/acisbg) in the $9.5-12 \mathrm{keV}$ energy range. We then scale this background map to match that expected in our observations by the ratio $C_{\text {data,9.5-12 }} / C_{\text {stow,9.5-12 }}$, where $C_{\text {data }}$ are the counts measured in the data while $C_{\text {stow }}$ are the counts detected in the stowed position.

We run the "wvdecomp" algorithm (Vikhlinin et al. 1998) on the data after subtracting the smoothed background maps, to search for extended sources on angular scales exceeding 30 arcsec. In four cases, we detect emission and use a circular region with a radius matched to the extent of the emission for flux estimates. In cases where no emission is detected, we use a circular region with a 2-3 arcmin radius to determine an upper limit $(2 \sigma)$ on the flux. We also measure a "control" flux estimate in the source-free zone to refine the background subtraction. In cases of positive signal, we subtract it from the source emission with a scaling based on the flux extraction area. The point source-free X-ray emission measurements obtained with Chandra are summarized in Table 4. The table content, including the characteristic quantities derived from the $\mathrm{X}$-ray luminosity, is equivalent to Table 2 for XMM-Newton data. All results based on these measurements are discussed in Section 4.

\subsection{Diffuse Group versus Central Galaxy Emission}

Given the faint and limited extent of the observed X-ray emission, one might question whether we are detecting genuine diffuse IGM emission from gas sitting in the group halo potential well, or simply emission from the halo associated to the central galaxy. In fact, the typical X-ray luminosity of elliptical galaxies at the redshift characterizing our groups, which appears consistent with the luminosity of local elliptical galaxies (Kim \& Fabbiano 2004; Lehmer et al. 2007), is of the order of $10^{40} \mathrm{erg} \mathrm{s}^{-1}$. This is an order of magnitude smaller than the threshold luminosity detectable with the exposure times of our observations, which is above $10^{41} \mathrm{erg} \mathrm{s}^{-1}$. Furthermore, based on the richness of the observed systems and the X-ray fluxes measurements or upper limits, it is pretty obvious that these emitting volumes are not embedded in a larger virialized structure, e.g., a galaxy cluster. Instead, luminosities above $10^{41} \mathrm{erg} \mathrm{s}^{-1}$ typically correspond to the IGM emission from the volume surrounding the central galaxy in modest groups (Duffy et al. 2008), which is in fact our assumption.

Clearly, the detected fluxes are not sufficient to determine the groups' masses from, e.g., the density and temperature profiles, as is usually done under the assumption of hydrostatic equilibrium. The group masses are instead obtained using scaling relations established independently for groups of similar, albeit slightly larger, X-ray luminosities by a weak lensing analysis (Leauthaud et al. 2010). This seems reasonable because, as already mentioned, according to Finoguenov et al. (2015), these relations also describe the weak lensing and clustering properties of lower mass groups well (with mass reaching below $10^{13} M_{\odot}$ ). We can finally check for rough consistency between the observed X-ray fluxes and the masses obtained from the above scaling arguments. In fact, the observed X-ray emission within $20 \mathrm{kpc}$ allows us to infer a gas temperature of $T=0.3 \mathrm{keV}$, necessary to produce emission in the X-ray spectral band, and a lower limit to the $\beta$ parameter for the surface brightness profile, $\beta=0.3$, i.e., the smallest value consistent with the absence of emission on scales larger than $20 \mathrm{kpc}$. Then, under the assumption of hydrostatic equilibrium, the implied mass within a $20 \mathrm{kpc}$ radius is $2 \times 10^{11} M_{\odot}$, with a corresponding dark matter density of $10^{4}$ times the critical value. Using the concentration-mass relation of Duffy et al. (2008), we obtain a virial mass of $10^{13} M_{\odot}$, consistent with the values obtained from the scaling relations, supporting our estimates. 
Note that in all cases, the reported extended X-ray emission covers the radii exceeding the effective radius of the central galaxy.

\section{RESULTS}

Our X-ray observations of galaxy groups in ZENS with $X M M-N e w t o n$ and Chandra enable us to begin the construction of a sample selected without an obvious preference on their $\mathrm{X}$-ray properties or dynamical state. The XMM-Newton data include observations of nine groups obtained through our observational programs and archival data of four fields, which happened to be in the proximity of four ZENS groups. The total set of 13 groups is listed in Table 1.

Of the nine ZENS groups observed with XMM-Newton through our programs, X-ray emission from the IGM was clearly detected in three cases (2PIGG_s1520, 2PIGG_s1571, 2PIGG_n1606), with a typical signal-to-noise $>4$, except for group 2PIGG_s1571, which has a much higher signal-to-noise of 46.87. Of the remaining six groups, X-ray observations include two low-significance detections likely contaminated by unassociated emission (2PIGG_s1614, 2PIGG_s1471), three upper limits, one of which is due to complications in the wavelet analysis associated with flare contamination causing a particularly high residual background (2PIGG_s1783), and one case of flare disruption (2PIGG_s1466). Flares affected the observations of three groups (2PIGG_s1614, 2PIGG_s1783, 2PIGG_s1799) compromising our ability to potentially detect their IGM diffuse X-ray emission (all programs were equally affected by flares). The archival data are included here for completeness because they unfortunately were not very useful in the end. In fact, the ZENS groups were only partially covered by the field of view and the flux was often dominated by a different source. For this reason, these groups were not included in any following analyses.

In comparison, of the 12 ZENS groups observed with Chandra and listed in Table 3, X-ray emission from the IGM was detected in a total of three cases (2PIGG_s1571, 2PIGG_n1320,2PIGG_n1746). One of these cases corresponds to 2PIGG_s1571 and, as for the case of XMM-Newton, it is detected with a high signal-to-noise ratio. The flux measured by the two telescopes is consistent within the reported statistical error. The remainder of the X-ray observations include a $2 \sigma$ detection (2PIGG_n1702) which is, however, not associated with our group while the rest are upper limits.

These results reveal the presence of diffuse intragroup gas inside the shallow potential well of small-scale groups with masses around $10^{13} M_{\odot}$. The X-ray emission is rather faint, with typical flux $\leqslant 10^{-13} \mathrm{erg} \mathrm{s}^{-1} \mathrm{~cm}^{-2}$, i.e., one order of magnitude fainter than typical ROSAT groups (RASS). In addition, for several groups, we were able to measure upper limits, which implies that we are mostly probing the upper envelope of the $\mathrm{X}$-ray emitting, optically selected ZENS groups. While this is likely due to diversity in X-ray luminosity of our groups at fixed mass, part of the effect is likely enhanced by uncertainties in the mass determination of the groups.

\subsection{X-Ray versus Optical Images}

In Figures 1-2, we show contours of wavelet-smoothed Xray emission maps for the ZENS groups, in which IGM diffuse emission has been successfully detected, overlaid with optical i-band images of the respective group fields. The groups include 2PIGG_s1520,2PIGG_s1571, 2PIGG_n1606 characterized by $\mathrm{S} / \mathrm{N}>6$ for XMM-Newton's observations, and 2PIGG_s1571, 2PIGG_n1320, 2PIGG_n1746 with S/N > 4 for Chandra observations. The contours indicate the surface brightness level of the X-ray emission in units of counts/s/ pixel starting on a log scale at -6.2 and -7.0 for XMM-Newton and Chandra, respectively, and increasing upward by increment of 0.2 (for 2PIGG_n1320 observed with Chandra the contours start one level higher, -6.8 , because there is no pixel with emission below that value). The pixel size is $4^{\prime \prime}$ and $\approx 1^{\prime \prime}$, for XMM-Newton and Chandra, respectively. Point sources were removed only for the case of XMM-Newton because they are easy to identify in Chandra's images (their contribution is removed when computing the X-ray diffuse emission). XMMNewton exposure maps used to normalize the emission are coadded pn and MOS values renormalized to Chandra ACIS-I on axis sensitivity, so total count rates are comparable in those units.

Member galaxies are identified with a small circle, blue in the case of satellites and red for central galaxies. In our ZENS analysis, whenever possible, the central galaxy was identified as the group member satisfying the following requirements: (1) to be the most massive galaxy in that group, accounting for errors in the stellar mass estimates, (2) to be close in position to the group center, and (3) to move with respect to the group bulk at a velocity that is within $1 \sigma$ of the group velocity dispersion. In roughly $40 \%$ of the groups, no member galaxy fulfilled all of the criteria above; in these cases, the central galaxy was simply defined as the most massive member in the group, albeit its velocity and position displayed offsets from the group mass and velocity centroid (see Carollo et al. 2013, for further details). In addition, in our ZENS work, we consistently assumed that the so-defined central galaxies identify the centers of the groups. This approach differs from the original 2PIGG procedure of Eke et al. who computed and used, as group center, a weighted average of the galaxy positions. Both of these approaches were developed prior to any knowledge of the groups' X-ray emission, which now allows for a sensible consistency check of either optical definitions of centers, and between group properties determined with optical and with $\mathrm{X}$-ray data. We also defined the dynamical state of the group depending on whether or not a central galaxy that satisfied all of the requirements described above could be found: groups in which no such galaxy existed were classified as unrelaxed, the others as relaxed.

We thus inspect the composite images in Figures 1-2. Furthermore, Table 5 lists the difference between the 2PIGG optically averaged and the X-ray centers of the groups (third column), defined as the peak in the X-ray emissivity map after removing the emission from detected point sources, as well as the distance of the group central galaxy to the 2PIGG (fourth column) and X-ray (fifth column) centers. The first three columns contain the groups' name and optical equatorial coordinates, while the last column indicates the groups' dynamical state, according to the optical data. The Table includes all groups for which a group center could be reliably determined from the X-ray data.

For three out of the five listed groups (2PIGG_s1520, 2PIGG_s1571, 2PIGG_n1320), the 2PIGG and X-ray centers coincide to within a few arcseconds. Furthermore, we note that for two of these three groups, namely 2PIGG_s1520 and 

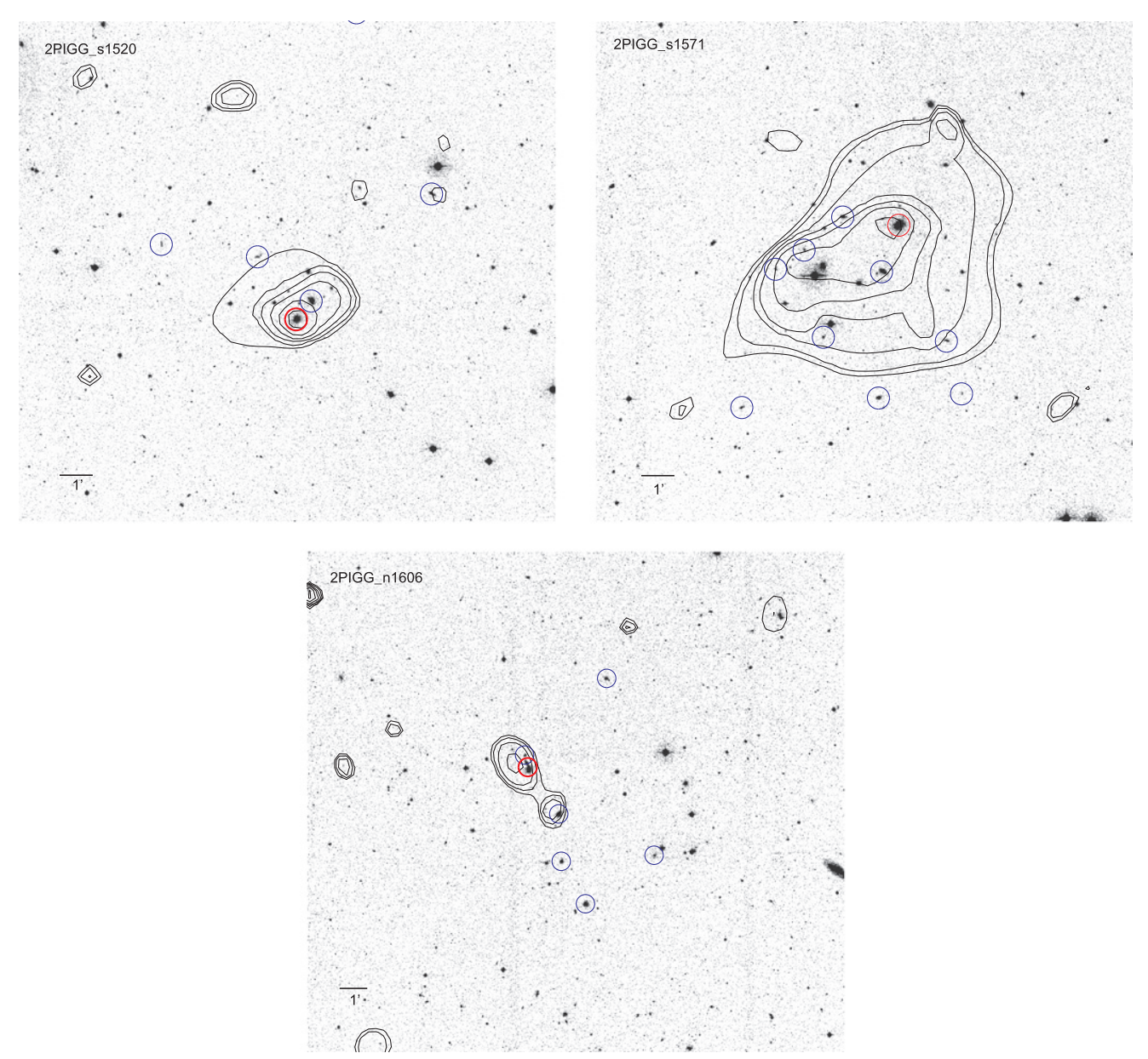

Figure 1. XMM-Newton measurement of the Diffuse Intragroup Medium X-ray emission from the ZENS groups 2PIGG_s1520 (top-left), 2PIGG_s1571 (top-right), and 2PIGG_n1606 (bottom), overlaid with optical i-band image. Black contours indicate the surface brightness level of X-ray emission on a log scale, starting at log $\left(\right.$ counts $\left.\mathrm{s}^{-1}\right)=-6.2$ and increasing upward by increments of 0.2 . X-ray point sources have been removed. Blue circles mark the galaxy members and the red circle marks the central galaxy as determined prior to knowledge of the X-ray emission.

2PIGG_s1571, the 2PIGG centers coincide with the central galaxy. The group 2PIGG_s1571 was classified in Paper I as a relaxed group, with the central galaxy identification indeed in agreement with both the 2PIGG-averaged center and the peak of the X-ray emission. This group thus provides reassurance that, in well-behaved systems, the center and central galaxy associations based on optical data are robust and physically motivated.

Things can be trickier, however, for more complex structures. The group 2PIGG_s1520 is classified as unrelaxed based on the optical data, but, nevertheless, there is good agreement between the X-ray peak and the 2PIGG-averaged center. On the other hand, while based on the optical data 2PIGG_1320 is classified as a relaxed group, the galaxy corresponding to the peak of the X-ray emission is substantially offset from the optically identified central galaxy of the group. In addition, according to the optical classification, the group 2PIGG_n1606 is relaxed, but its diffuse emission is clearly double peaked, which is suggestive of a merger event. In 2PIGG_n1606, the offset between the optical and X-ray centers as well as optical center and central galaxy, is much larger than the separation between X-ray center and central galaxy. This is consistent with the X-ray picture that the system is undergoing a merger, the system is unrelaxed, and the X-ray emission traces the largest of the merging structures. Finally, the group
2PIGG_n1746, characterized by the smallest discrepancy, is classified as relaxed from the optical. 2PIGG_n1746 is characterized by a mild separation between the 2PIGG and the X-ray centers, though the X-ray center is closer to the central galaxy than to the optical center. Therefore, this group is also likely to be dynamically unrelaxed in spite of the optical classification, as in the case of 2PIGG_n1606 discussed above.

These examples illustrate that there is substantial difficulty in identifying group centers from optical data alone, and, most likely, also a substantial diversity in group properties and dynamical conditions. While the optical and X-ray data are often consistent, in several cases, important discrepancies become apparent and complementary X-ray observations become important for a complete picture (see also George et al. 2012). It is the combination of high-quality optical data and X-ray observations that reveals subtle properties of the dynamical state of groups, which would be missed by an analysis based purely on either data set alone. This information is important in studies of galaxy properties and evolution as a function of group environment, which aim at establishing the physical processes and conditions responsible for triggering evolutionary mechanisms. It is obvious that misclassifications of the group center and/or dynamical state, introduce noise in the observed relations, which can be amended with X-ray data. 


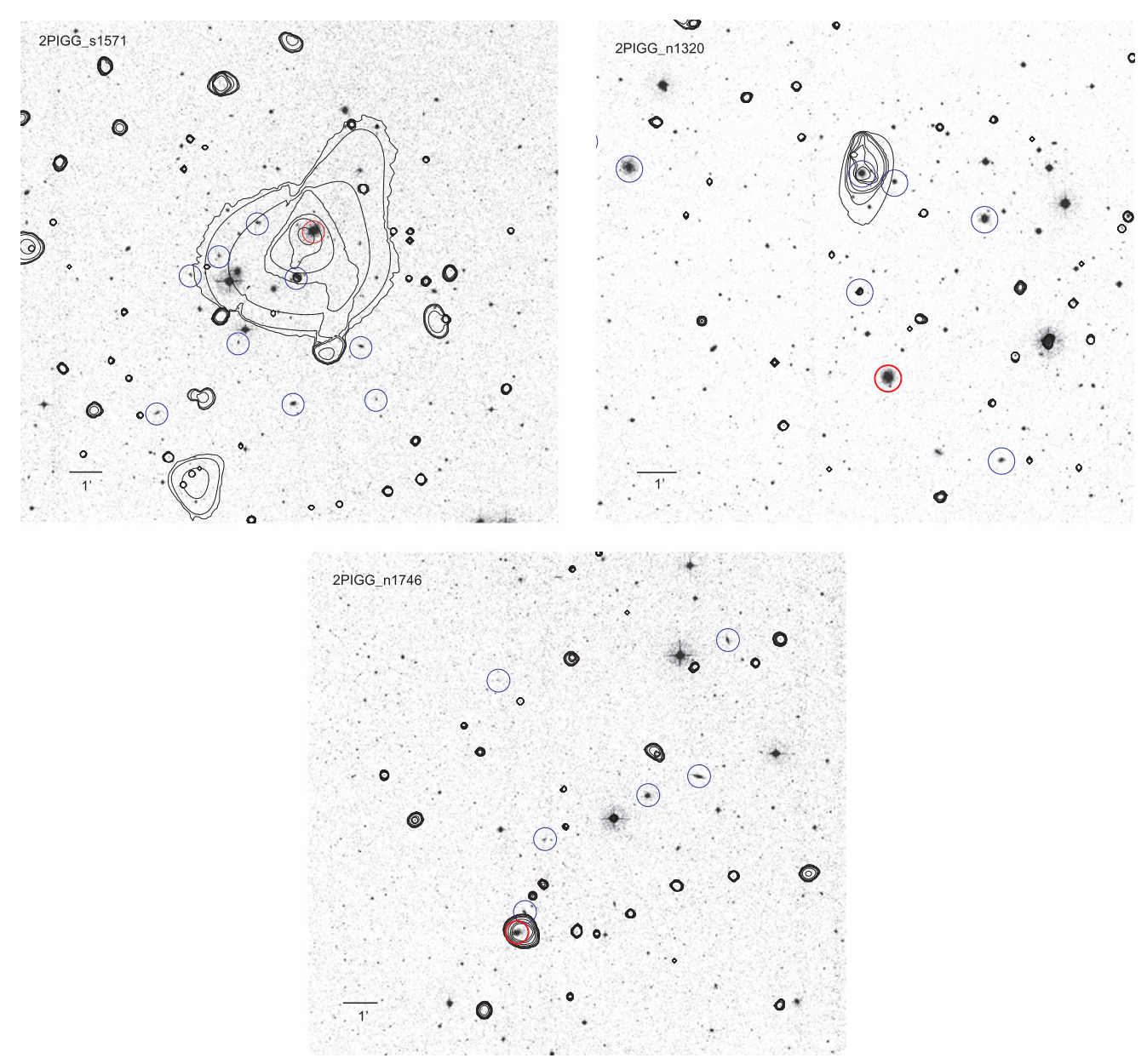

Figure 2. Chandra measurements of the Diffuse Intragroup Medium X-ray emission from the ZENS groups 2PIGG_s1571 (top-left), 2PIGG_n1320 (top-right), and 2PIGG_n1746 (bottom), overlaid with optical the i-band image. Black contours indicate the surface brightness level of X-ray emission on a log scale, starting at log $\left(\right.$ counts $\left.\mathrm{s}^{-1}\right)=-7.0$, and increasing upward by an increment of 0.2 (except for 2PIGG_n1320 the contours start one level higher, -6.8 , because there is no pixel with emission below that value). X-ray point sources have not been removed. Blue circles mark the galaxy members and the red circle marks the central galaxy as determined prior to knowledge of the X-ray emission.

Table 5

Optical versus X-Ray Centers

\begin{tabular}{|c|c|c|c|c|c|c|}
\hline Group & $\begin{array}{l}\text { R.A. } \\
\text { (J2000) }\end{array}$ & $\begin{array}{l}\text { decl. } \\
(\mathrm{J} 2000)\end{array}$ & $\begin{array}{c}\Delta \alpha_{\text {Opt-Xray }} \\
\text { (") }\end{array}$ & $\Delta \alpha_{\text {Opt-CG }}$ & $\begin{array}{c}\Delta \alpha_{\mathrm{CG}-\mathrm{Xray}} \\
\text { (") }\end{array}$ & State $^{\mathrm{a}}$ \\
\hline 2PIGG_s1520 & 00:02:01.79 & $-34: 52: 55.5$ & 3.3 & 0.23 & 3.3 & $\mathrm{U}$ \\
\hline 2PIGG_s1571 & $02: 37: 04.33$ & $-25: 23: 34.3$ & 7.4 & 0.24 & 7.3 & $\mathrm{R}$ \\
\hline 2PIGG_n1606 & $10: 38: 36.50$ & 01:46:01.2 & 280 & 268 & 36 & $\mathrm{R}$ \\
\hline 2PIGG_n1320 & $10: 17: 55.04$ & $-01: 22: 53.4$ & 1.8 & 312 & 310 & $\mathrm{R}$ \\
\hline 2PIGG_n1746 & $14: 40: 20.07$ & $-03: 45: 56.2$ & 36 & 38 & 10 & $\mathrm{R}$ \\
\hline
\end{tabular}

Note.

${ }^{\mathrm{a}} \mathrm{R}=$ relaxed, $\mathrm{U}=$ unrelaxed.

\section{2. $L_{X}$ versus $M$ Diagram and Mass Determinations}

In the bottom left panel of Figure 3, we plot the group mass as a function of the IGM X-ray luminosity. Data points for groups in which diffuse $\mathrm{X}$-ray emission has been detected are shown for XMM-Newton and Chandra as blue circles and red pentagons, respectively. The remainder of the groups are represented by upper limit symbols, with the same color code as above. Open and solid style indicates relaxed and unrelaxed systems, respectively, while horizontal bars correspond to statistical errors in the X-ray luminosity reported in Tables 1 and 3. The group mass, $M_{\mathrm{opt}}$, also listed in the above Tables, is provided by the ZENS catalog and is based on the optical luminosity of the group member galaxies. It is computed from the total optical luminosity of the group $L_{\text {Group }}$, using the massto-light ratio (see Eke et al. 2004a, for details)

$$
\log \left(Y_{b_{j}}\right)=2.28+0.4 \tanh \left\{1.9\left[\log \left(L_{\text {Group }}\right)-10.6\right]\right\} .
$$

Here $L_{\text {Group }}$ is calculated by summing up the luminosities of the individual group members, correcting for both the 2dFGRS spectroscopic incompleteness and for the fraction of galaxies that fall below the 2dFGRS magnitude limit of $b_{j}=19.45$. We estimated in Carollo et al. (2013) that the typical uncertainty in 

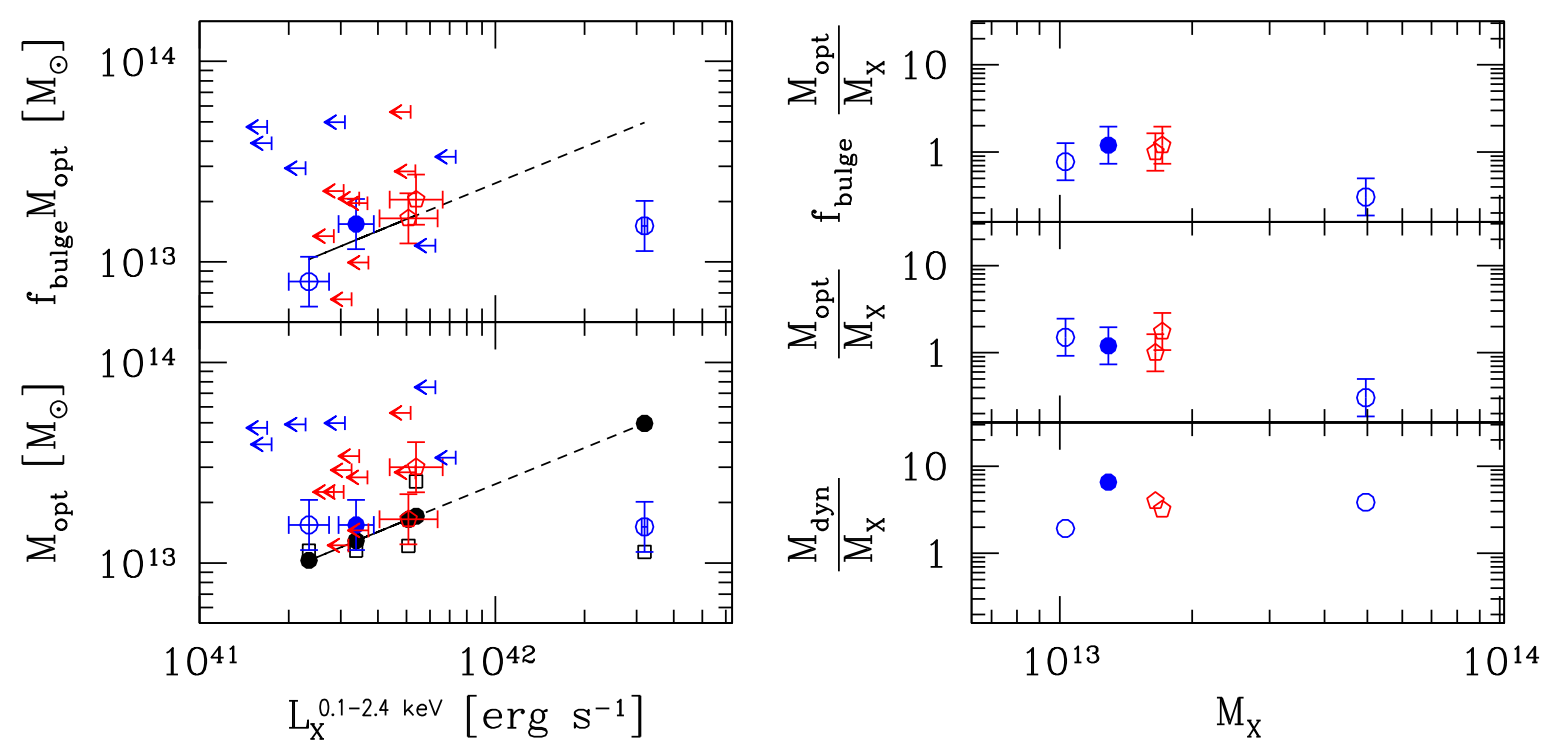

Figure 3. Left: optical (bottom) and bulge-based optical ( $\equiv f_{\text {bulge }} \times M_{\text {opt }}$ ) (top) group mass vs. X-ray luminosity from the diffuse intragroup medium. Measurements from XMM-Newton (blue circles) and Chandra (red pentagons) with signal-to-noise better than $\sim 4$ are shown together with upper limits for the respective instruments. Open and solid symbols denote relaxed and unrelaxed systems, respectively. Vertical bars correspond to $30 \%$ mass errors in the optical mass determination. Horizontal bars correspond to statistical errors in the X-ray luminosity as reported in Tables 13 . The group mass for the ZENS sample is obtained from the groups optical luminosity and is given in the same tables. For comparison, the black solid circles connected by a dashed line indicate the position of the groups in the diagram if their mass is derived from the X-ray luminosity using the $L_{\mathrm{X}}-M_{\mathrm{X}}$ relation established from the joint analysis of $\mathrm{X}$-ray and weak lensing for groups in COSMOS (Leauthaud et al. 2010). Right: dynamical (bottom), optical (middle), and bulge-based optical group mass estimates divided by the X-ray mass. Data from $X M M$-Newton (blue circles) and Chandra (red pentagons) with signal-to-noise better than four are shown. Open and solid symbols indicate relaxed and unrelaxed systems respectively. Error bars include errors associated with the X-ray mass determination reported in Tables 2 and 4, and the 30\% mass estimate for the optical mass (see Carollo et al. 2013).

the thus derived halo masses is of the order of 0.3 dex. This estimate takes into account the effect of interlopers in the group members as well as the uncertainties in the conversion of the galaxy luminosity function to a halo mass. In the following, we assume this value as the typical error for $M_{\mathrm{opt}}$.

In order to compare with predictions from scaling relations, in the same plot, we also present the black solid circles connected by a dashed line. They indicate the position of the groups in the diagram if we derive their mass, $M_{\mathrm{X}}$, from the $\mathrm{X}$-ray luminosity using the $L_{\mathrm{X}}-M_{\mathrm{X}}$ relation in Leauthaud et al. (2010). This relation is based on a joint analysis of X-ray and weak lensing of groups in the COSMOS survey. The plot shows that our groups depart significantly from the scaling relation inferred from the X-ray-selected groups. Except for 2PIGG_s1571, our ZENS groups appear to be under luminous for their stellar content.

The discrepancy is further illustrated in the middle-right panel, where the analysis is now restricted to groups with detected X-ray emission only. In this panel, the optical to X-ray mass ratio is plotted as a function of the $\mathrm{X}$-ray mass, $M_{\mathrm{X}}$. There is a potential relative bias between the two estimates, as the optical masses appear systematically larger than the X-ray masses with the single exception of 2PIGG_s1571, which is, however, unusually bright. To quantify this possibility, we compute a mean ratio, excluding somewhat arbitrarily 2PIGG_s1571, and find $\left\langle M_{\text {opt }} / M_{\mathrm{X}}\right\rangle-1 \approx 0.36 \pm 0.3$, i.e., a potential bias of almost $40 \%$, albeit with a modest significance. When we compare the dynamical mass estimates (i.e., Connelly et al. 2012)

$$
M_{\mathrm{dyn}}=\frac{3 \sigma_{v}^{2} R_{200}}{G_{N}}
$$

to the X-ray mass values (bottom right panel), we find an even larger bias, in particular, $\left\langle M_{\mathrm{dyn}} / M_{\mathrm{X}}\right\rangle-1 \approx 3$. This is consistent with the roughly 0.2 dex difference between the $M_{\text {dyn }}$ and $M_{\text {opt }}$ found in the analysis of Carollo et al. (2013). The larger bias in $M_{\mathrm{dyn}}$ is mostly due to large uncertainties associated with the determination of velocity dispersions with respect to the group center of mass when the measurements are based on just a few member galaxies.

In addition, it is well known that if the group is dynamically unrelaxed, the velocity dispersion will lead to the overestimation of the actual mass of the group. However, in our limited sample, there seems to be no indication that unrelaxed groups (solid symbols) are more under luminous than relaxed ones (open symbols). Also, inspection of the individual group properties shows that the $\mathrm{X}$-ray under-luminous groups span a substantial range in terms of bulge fraction of the central galaxy, which suggests that the "under luminosity" reported in the left panel of Figure 3 may not be simply related to a recent formation of the group. Instead, we have found a slight correlation between the optical mass bias and the bulge fraction, in the sense that the bias of the optical versus X-ray mass determination seems to increase toward a smaller bulge fraction. The correlation has an almost $3 \sigma$ significance (the ratio of the slope to the statistical error) for objects above $10^{13} M_{\odot}$ (but is reduced when the bright 2 PIGG_s1571 is included). The ratio of the optical-to-X-ray group mass times the bulge fraction is plotted as a function of the X-ray mass in the top-left panel of Figure 3. According to our analysis $\left\langle f_{\text {bulge }} M_{\text {opt }} / M_{\mathrm{X}}\right\rangle-1 \approx 0.04 \pm 0.17$, about one and two orders of magnitude smaller than the optical and dynamical mass estimates, respectively. Thus the group stellar mass in the bulge component might be a better proxy to the group mass than $M_{\mathrm{opt}}$ 


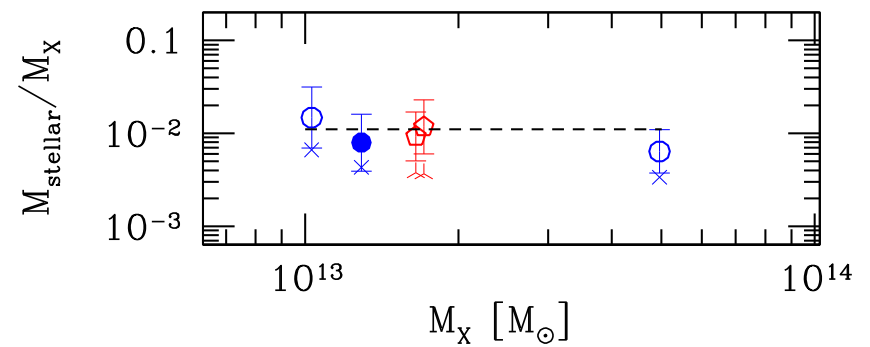

Figure 4. Stellar-to-total mass ratio as a function of the X-ray mass, where the total mass is the X-ray mass obtained using the scaling relations in Leauthaud et al. (2010). Blue and red symbols indicate X-ray data from XMM-Newton and Chandra, respectively, while open and solid symbols indicate relaxed and unrelaxed systems respectively. Crosses correspond to the stellar mass fraction contributed by the group's most massive galaxy and are located below the circle or pentagon symbol of the corresponding group. The dashed line indicates the median value of 0.011. Only groups with detected diffuse X-ray emission are shown. Upper limits do not contribute additional information because they mostly crowd above the median bar of the top panel. Error bars include errors associated with the X-ray mass determination reported in Tables 2 and 4, and errors based on the upper limits of the best stellar mass estimate given in Carollo et al. (2013).

based on the total optical emission. Of course, our result should be tested using a group data set. Note that similar results were also found in Andreon (2012), although at considerably larger mass scales.

\subsection{Stellar Mass Fraction}

In Figure 4, we plot the stellar-to-total mass ratio as a function of the X-ray mass, $M_{\mathrm{X}}$, where the total mass is $M_{\mathrm{X}}$. The stellar mass is conventionally computed using galaxies with masses down to $10^{10} M_{\odot}$ (Leauthaud et al. 2012), where the ZENS catalog is complete for any spectral type. Given that we do not correct the total stellar mass for the contribution of galaxies with $M<10^{10} M_{\odot}$, our values of the stellar mass fraction should be treated as lower limits on the true fraction. In Figure 4, blue and red symbols indicate X-ray data from XMMNewton and Chandra, respectively, while open and solid symbols indicate relaxed and unrelaxed systems respectively. Only groups with detected X-ray emission are shown, and upper limits are not plotted because they do not contribute additional information since they mostly crowd above the median values. The latter is indicated by a dashed line, which corresponds to a value of 0.011 .

Four out of five of the stellar fractions computed are within $1 \sigma$ of the median value, with the exception of 2PIGG_1571, which is about $1.7 \sigma$ lower than the median value. The median of 0.011 is consistent with results obtained by Connelly et al. (2012) for optically selected groups who determined the group masses using the X-ray luminosity together with the calibration of Leauthaud et al. (2010). The median value found is also consistent with results of Balogh et al. (2011), at a similar sample size, who determine the group masses using X-ray profiles. Our fractions are lower than the values reported in Giodini et al. (2009), who also used X-ray-based group masses, but used a different initial mass function and measured the stellar mass fraction within $R_{500}$, as opposed to the $R_{200}$ as in our case. They are also lower than reported by Gonzalez et al. (2007) who instead used dynamical masses and took into account the contribution from intracluster light.

For comparison, we also plot the stellar mass fraction contributed by the group's most massive galaxy. It is indicated

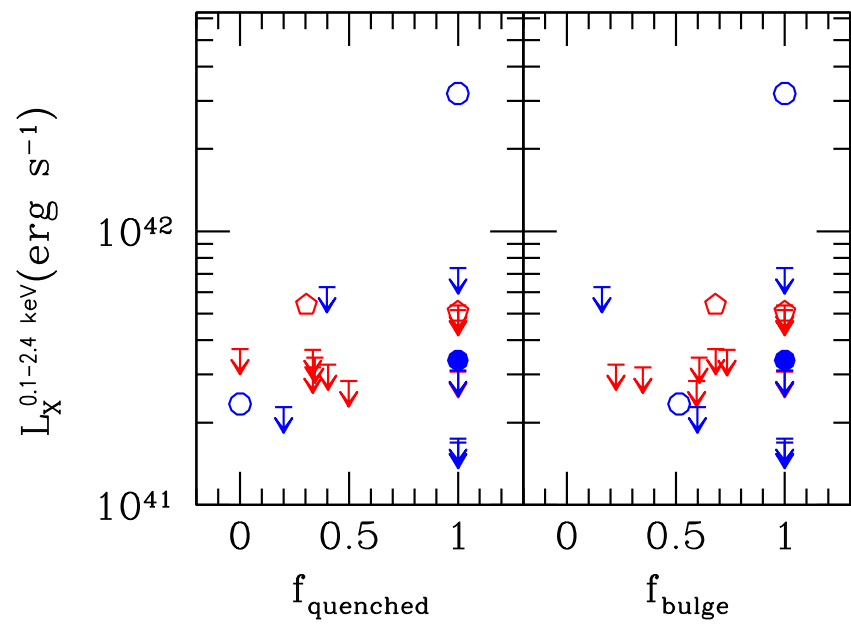

Figure 5. Group X-ray luminosity as a function of the fraction of group galaxies with quiescent star formation (left) and the fraction of bulge stellar mass of the group member galaxies (right). Quantities are computed from galaxies with masses above $10{ }^{10} M_{\odot}$, where the ZENS catalog is complete. The star formation for individual galaxies is determined through photometric and spectroscopic measurements and proper correction for incompleteness; the bulge component is determined using a bulge-disk decomposition based on both I- and B-band photometric data (Cibinel et al. 2013b). Data from XMMNewton (blue circles) and Chandra (red pentagons) for groups with detected diffuse X-ray emission are shown (circles and pentagons) together with upper limits for the respective instruments. Open and solid symbols indicate relaxed and unrelaxed systems respectively.

with a blue or red cross below the circle or pentagon symbol of the corresponding group. The plot shows that the most massive galaxy typically contains between $30 \%$ and $50 \%$ of the total stellar mass fraction in the group.

\section{4. $L_{X}$ versus Global Galaxy Properties}

As a first attempt to investigate whether either the diffuse $\mathrm{X}$-ray emission or the galaxy properties or both show hints for a substantial interaction between the IGM and the group galaxies, we searched for correlations between the X-ray properties of the groups, i.e., their luminosity, and the general properties of the group member galaxies as a whole.

In Figure 5, we show two examples of correlation that could, in principle, reveal such an effect. In the left panel of Figure 5, for each group, we plot the X-ray luminosity from the diffuse intragroup medium against the fraction of group galaxies that are classified as quenched in Cibinel et al. (2013b). These galaxies were identified as such from the absence of emission lines in the 2dFGRS spectra and their location on NUV-optical color-color diagrams optimized to disentangle dusty, red galaxies from truly passive systems (see Cibinel et al. 2013b, for details). In addition, in the right panel of Figure 5, the X-ray luminosity is plotted against $f_{\text {bulge. }}$ As in previous cases, in both figures, data from XMM-Newton (blue circles) and Chandra (red pentagons) with detected diffuse X-ray emission are shown, together with upper limits for the respective instruments.

The $L_{\mathrm{X}}$ versus quenched fraction could contain information about the role of the IGM in quenching the star formation in group galaxies, while, conversely, the spheroid component, which correlates with black hole mass, could reveal the impact of AGN activity on the IGM. However, we see no apparent correlation in either plot using the current data. The small size of the sample and the numerous upper limits compared to 
$\mathrm{X}$-ray detections are clearly a limitation in this investigation. A larger sample, at fixed group halo mass as opposed to one with a larger range in X-ray luminosity, would be most useful to disentangle effects associated with group halo mass, to separately investigate trends for central and satellite galaxy samples, and for satellites as a function of group-centric distance-both recognized key environmental parameters (Weinmann et al. 2006, 2008; Carollo et al. 2013).

\section{CONCLUSIONS}

We have conducted a pilot program of X-ray observations with the XMM-Newton and Chandra telescopes of a small subset of optically selected groups belonging to the sample of 141 groups of the ZENS. Observations with XMM-Newton were carried out for nine groups during three observing cycles. With the addition of archival data for four groups, they amount to a total of 13 group observations. About one-third of the data was lost due to flares. X-ray emission from the IGM was successfully detected for three groups with $\mathrm{S} / \mathrm{N} \gtrsim 6$. The Chandra data were collected during Cycle 11. A total of 12 ZENS groups were observed and diffuse X-ray emission from the IGM was successfully detected for three groups with $\mathrm{S} / \mathrm{N} \gtrsim 4$.

The target groups have been selected to be in a narrow mass scale about $10^{13} M_{\odot}$, and at redshift $z=0.05-0.0585$. The detections reveal a typical X-ray flux in the energy band $0.5-2 \mathrm{keV}$ of $\leqslant 10^{-13} \mathrm{erg} \mathrm{s}^{-1} \mathrm{~cm}^{-2}$, which is one order of magnitude fainter than typical ROSAT groups (RASS). However, for many groups, we were able to obtain only upper limits, indicating that despite uncertainties in the mass determination of the groups, our detections are likely probing the upper envelope of the X-ray emitting, optically selected ZENS groups.

The main results of this exploratory analysis can be summarized as follows.

1. Small groups such as those probed by the ZENS survey are characterized by a large diversity of properties and dynamical conditions. The X-ray data, in particular, maps of the IGM, reveal features that would be missed by the analysis of the optical data alone. They thus provide important complementary information to optical data, which is necessary particularly for a robust classification of the dynamical conditions of the group.

2. Our investigation of the X-ray luminosity versus group mass indicates that our optically selected ZENS groups may be under luminous with respect to the prediction from scaling relations characterizing X-ray selected groups Leauthaud et al. (2010), as they crowd almost exclusively the space to the left of the $M_{\mathrm{X}}$ versus $L_{\mathrm{X}}$ line corresponding to the said scaling relation, the resulting asymmetry being even stronger than reported in Connelly et al. (2012).

3. The mass determination based on the total optical luminosity of the groups is in better agreement with the mass determination based on X-ray emission than the dynamical mass estimates. The optical masses, however, appear to be higher than those from X-ray measurements by almost $40 \%$. Given the relatively large uncertainty on the mass determinations, this result is only slightly significant. We find, however, that a mass determination based on the luminosity of the bulge component of the member galaxies, rather than the total luminosity, reduces the potential bias with respect to the X-ray mass estimates by almost one to two orders of magnitude compared to the values inferred from the total optical luminosity and dynamical mass estimates, respectively (consistent with Andreon 2012, at larger mass scales). Note that the dynamical mass estimates could also be improved with refined determinations of the group member galaxies' velocity dispersion as proposed recently in Erfanianfar et al. (2013).

4. The group stellar mass fraction, obtained from the ratio of the group's total stellar mass and the total group mass determined from the X-ray emission, has a median value of 0.011 . This is consistent with the work of Connelly et al. (2012). The contribution to the stellar mass fraction from the most massive galaxies ranges between $30 \%$ and $50 \%$.

It is clear that the small number of diffuse X-ray measurements for $\sim 10^{13} M_{\odot}$ groups limits at the moment our ability to robustly probe the effects of the interplay between the IGM and the member galaxies at this mass scale that is likely very relevant for environmentally driven galactic evolution. However, this pilot program demonstrates the potential return and importance of conducting a similar X-ray study on a group sample that is large enough to enable a statistically robust investigation of this crucial and yet unexplored issue in galaxy evolution.

We are very thankful to an anonymous referee for a very careful reading and constructive comments.

\section{REFERENCES}

Andreon, S. 2012, A\&A, 548, A83

Balogh, M. L., Mazzotta, P., Bower, R. G., et al. 2011, MNRAS, 412, 947

Barnes, J. E. 1990a, Natur, 344, 379

Barnes, J. E. 1990b, MNRAS, 333, 481

Barnes, J. E., \& Hernquist, L. E. 1991, ApJL, 370, L65

Bernet, M. L., Miniati, F., \& Lilly, S. J. 2010, ApJ, 711, 380

Bernet, M. L., Miniati, F., \& Lilly, S. J. 2012, ApJ, 761, 144

Bernet, M. L., Miniati, F., Lilly, S. J., Kronberg, P. P., \& Dessauges-Zavadsky, M. 2008, 454, 302

Bielby, R. M., Finoguenov, A., Tanaka, M., et al. 2010, A\&A, 523, A66 Borgani, S., Finoguenov, A., Kay, S., et al. 2005, MNRAS, 361, 233 Bower, R. G., Benson, A. J., Malbon, R., et al. 2006, MNRAS, 370, 645 Bower, R. G., McCarthy, I. G., Benson, A. J., et al. 2008, MNRAS, 390, 1399 Bruzual, G., \& Charlot, S. 2003, MNRAS, 344, 1000

Burns, J. O., Ledlow, M. J., Loken, C., et al. 1996, ApJL, 467, L49 Cappelluti, N., Ranalli, P., Roncarelli, M., et al. 2012, MNRAS, 427, 651

Carollo, C. M., Cibinel, A., Lilly, S. J., et al. 2013, ApJ, 776, 71 Carollo, C. M., Cibinel, A., Lilly, S. J., et al. 2014, arXiv:1402.1172 Cavaliere, A., Menci, N., \& Tozzi, P. 1998, ApJ, 501, 493

Cibinel, A., Carollo, C. M., Lilly, S. J., et al. 2013a, ApJ, 776, 72 Cibinel, A., Carollo, C. M., Lilly, S. J., et al. 2013b, ApJ, 777, 116 Colless, M., Dalton, G., Maddox, S., et al. 2001, MNRAS, 328, 1039 Connelly, J. L., Wilman, D. J., Finoguenov, A., et al. 2012, ApJ, 756, 139 Croton, D., Springel, V., White, S. D. M., et al. 2006, MNRAS, 367, 864 Donahue, M., Scharf, C. A., Mack, J., et al. 2002, ApJ, 569, 689 Duffy, A. R., Joop, S., Kay, S. T., et al. 2008, MNRAS, 390, L64 Ebeling, H., Voges, W., \& B'oringer, H. 1994, ApJ, 436, 44 Ekcmiller, H. J., Hudson, D. S., \& Reiprich, T. H. 2012, A\&A, 535, A105 Eke, V. R., Baugh, C. M., Cole, S., et al. 2004a, MNRAS, 348, 886 Eke, V. R., Baugh, C. M., Cole, S., et al. 2005, MNRAS, 362, 1233 Eke, V. R., Frenk, C. S., Bauch, C. M., et al. 2004b, MNRAS, 355, 769 Erfanianfar, V., Finoguenov, A., Tanaka, M., et al. 2013, ApJ, 765, 117 Feldmann, R., Carollo, C. M., Mayer, L., et al. 2010, ApJ, 709, 218 Finoguenov, A., Arnaud, M., \& David, L. P. 2001, ApJ, 555, 191 Finoguenov, A., Connelly, J. L., Parker, L. C., et al. 2009, ApJ, 704, 564 Finoguenov, A., Guzzo, L., Hasinger, G., et al. 2007, ApJS, 172, 182 
Finoguenov, A., Tanaka, M., Cooper, M., et al. 2015, A\&A, 576, A130 Fukugita, M., Hogan, C. J., \& Peebles, P. J. E. 1998, ApJ, 503, 518

George, M. R., Leauthaud, A., Bundy, K., et al. 2012, ApJ, 757, 2

Gilbank, D. G., Bower, R. G., Castander, F. J., \& Ziegler, B. L. 2004, MNRAS, 348, 551

Giodini, S., Finoguenov, A., Pierini, D., et al. 2012, A\&A, 538, A104

Giodini, S., Pierini, D., Finoguenov, A., et al. 2009, ApJ, 703, 982

Giodini, S., Smolcic, V., Finoguenov, A., et al. 2010, ApJ, 714, 218

Gonzalez, A. H., Zaritsky, D., \& Zabludoff, A. I. 2007, ApJ, 666, 147

Heldson, S. F., \& Ponman, T. J. 2000, MNRAS, 315, 356

Hickox, R. C., \& Markevitch, M. 2006, ApJ, 645, 95

Kawata, D., \& Mulchaey., J. 2008, ApJL, 672, L103

Kim, D. W., \& Fabbiano, G. 2004, ApJ, 611, 846

Knobel, C., Lilly, S. J., Kovac, K., et al. 2012, arXiv:1211.5607

Komatsu, E., Dunkley, J., Nolta, M. R., et al. 2009, ApJS, 180, 330

Leauthaud, A., Finoguenov, A., Kenib, J.-P., et al. 2010, ApJ, 709, 97

Leauthaud, A., George, M. R., Behroozi, P. S., et al. 2012, ApJ, 746, 95

Lehmer, B. D., Brandt, W. N., Alexander, D. M., et al. 2007, ApJ, 657, 681

Lloyd-Davis, E. J., Ponman, T. J., \& Cannon, D. B. 2000, MNRAS, 315, 689

Lubin, L. M., Mulchaey, J. S., \& Ponman, T. J. 2004, ApJL, 601, L9

Mahdavi, A., Böheringer, H., Geller, M. J., \& Ramella, M. 1997, ApJ, 483, 68

Mahdavi, A., Böheringer, H., Geller, M. J., \& Ramella, M. 2000, ApJ, 534, 114

Mahdavi, A., Finoguenov, A., Böhringer, H., Geller, M. J., \& Henry, J. P. 2005, ApJ, 622, 187

Markevitch, M. 1998, ApJ, 504, 27

Mayer, L., Governato, F., Colpi, M., et al. 2001, ApJ, 559, 754

McCarthy, I. G., Babul, A., Bower, R. G., \& Balogh, M. L. 2008, MNRAS, 386, 1309

McCarthy, I. G., Schaye, J., Ponman, T. J., et al. 2010, MNRAS, 406, 822

McGee, S. L., Balogh, M. L., Wilman, D. J., et al. 2011, MNRAS, 413, 996

McIntosh, D. H., Guo, Y., Hertzberg, J., et al. 2008, MNRAS, 388, 1537

Mihos, C. 2003, in Clusters of Galaxies: Probes of Cosmological Structure and Galaxy Evolution, Vol. 3, ed. J. S. Mulchaey, A. Dressler, \& A. Oemler (Cambridge: Cambridge Univ. Press), 2003

Mulchaey, J. 2000, ARA\&A, 38, 289
Mulchaey, J., Davis, D. S., Mushotzky, R. F., \& Burstein, D. 2003, ApJ, 145,39

Osmond, J. P. F., \& Ponman, T. J. 2004, MNRAS, 350, 1511

O’Sullivan, E., Kolokythas, K., Raychaudhury, S., Vrtilek, J. M., \& Kantharia, N. 2014, arXiv:1402.4676

Paturel, G., Petit, C., Prugniel, P., et al. 2003, A\&A, 412, 45

Peng, Y.-j., Lilly, S. J., Renzini, A., \& Carollo, M. 2012, ApJ, 757, 4

Poggianti, B. M., Desai, V., Finn, R., et al. 2008, ApJ, 684, 888

Ponman, T. J., Bourner, P. D. J., Ebeling, H., \& B'oringer, H. 1996, MNRAS, 283, 690

Ponman, T. J., Cannon, D. B., \& Navarro, J. F. 1999, Natur, 397, 135

Ponman, T. J., Sanderson, A. J. R., \& Finoguenov, A. 2003, MNRAS, 343, 331

Popesso, P., Biviano, A., Böringer, H., \& Romaniello, M. 2007, A\&A, 461, 397

Prat, G. W., Arnaud, M., Piffaretti, R., et al. 2010, A\&A, 511, A85

Rasmussen, J., \& Ponman, T. J. 2007, MNRAS, 380, 1554

Rasmussen, J., \& Ponman, T. J. 2009, MNRAS, 399, 239

Rasmussen, J., Ponman, T. J., Mulchaey, J. S., Miles, T. A., \& Raychaudhury, S. 2006, MNRAS, 373, 653

Robotham, A. S. G., Liske, J., Driver, S. P., et al. 2013, MNRAS, 431, 167

Silverman, J. D., Miniati, F., Finoguenov, A., et al. 2014, ApJ, 780, 67

Skibba, R. A. 2009, MNRAS, 392, 1467

Tal, T., Dekel, A., Oesch, P., et al. 2014, arXiv:1401.2984

Tanaka, M., Finoguenov, A., Mirkazemi, M., et al. 2013, PASJ, 65, 17

van den Bosch, F. C., Aquino, D., Yang, X., et al. 2008, MNRAS, 387, 79

Vikhlinin, A., McNamara, B. R., Forman, W., et al. 1998, ApJ, 502, 558

Voit, G. M., Kay, S. T., \& Bryan, G. L. 2005, MNRAS, 364, 909

Weinmann, S. M., van den Bosch, F. C., Yang, X., et al. 2006, MNRAS, 372, 1161

Weinmann, S. M., Kauffmann, G., van den Bosch, F. C., et al. 2008, MNRAS, 394, 1213

Wetzel, A. R., Tinker, J. L., Conroy, C., \& van den Bosch, F. C. 2013, MNRAS, 432, 336

York, D., Adelman, J., Anderson, J. E., Jr., et al. 2000, AJ, 120, 1579 